Article

\title{
The Complete Mitochondrial Genome of Mindarus keteleerifoliae (Insecta: Hemiptera: Aphididae) and Comparison with Other Aphididae Insects
}

\author{
Yuan Wang ${ }^{1,2}$, Jing Chen ${ }^{1}$, Li-Yun Jiang ${ }^{1}$ and Ge-Xia Qiao ${ }^{1, *}$ \\ Received: 8 October 2015; Accepted: 8 December 2015; Published: 17 December 2015 \\ Academic Editor: Lee A. Bulla \\ 1 Key Laboratory of Zoological Systematics and Evolution, Institute of Zoology, \\ Chinese Academy of Sciences, Beijing 100101, China; wangyuan0330@163.com (Y.W.); \\ chenjing@ioz.ac.cn (J.C.); jiangliyun@ioz.ac.cn (L.-Y.J.) \\ 2 College of Life Sciences, University of Chinese Academy of Sciences, Beijing 100049, China \\ * Correspondence: qiaogx@ioz.ac.cn; Tel.: +86-10-6480-7133
}

\begin{abstract}
The mitogenome of Mindarus keteleerifoliae Zhang (Hemiptera: Aphididae) is a 15,199 bp circular molecule. The gene order and orientation of M. keteleerifoliae is similarly arranged to that of the ancestral insect of other aphid mitogenomes, and, a tRNA isomerism event maybe identified in the mitogenome of M. keteleerifoliae. The $t R N A$-Trp gene is coded in the J-strand and the same sequence in the $N$-strand codes for the $t R N A$-Ser gene. A similar phenomenon was also found in the mitogenome of Eriosoma lanigerum. However, whether tRNA isomers in aphids exist requires further study. Phylogenetic analyses, using all available protein-coding genes, support Mindarinae as the basal position of Aphididae. Two tribes of Aphidinae were recovered with high statistical significance. Characteristics of the M. keteleerifoliae mitogenome revealed distinct mitogenome structures and provided abundant phylogenetic signals, thus advancing our understanding of insect mitogenomic architecture and evolution. But, because only eight complete aphid mitogenomes, including M. keteleerifoliae, were published, future studies with larger taxon sampling sizes are necessary.
\end{abstract}

Keywords: aphid; Mindarus keteleerifoliae Zhang; mitogenomes; tRNA isomerism; phylogenetic relationships

\section{Introduction}

Generally, the insect mitochondrial genome (mitogenome) consists of a circular, two-stranded genome, 14,000-19,000 bp in length, which contains 37genes, including 13 protein coding genes (PCGs), cytochrome coxidase subunits 1-3 (cox1, $\operatorname{cox} 2$ and $\operatorname{cox} 3)$, cytochrome $b(c o b)$, subunits 6 and 8 of the ATPase (atp6 and atp8), NADH dehydrogenase subunits 1-6 and 4L (nad1-6 and nad4L), two ribosomal RNA genes encoding the large and small subunit rRNAs ( $r r n L$ and $r r n S$ ) and 22 transfer RNA (tRNA) genes. An A+T-rich region, also named the control region, containing initiation sites for transcription and replication was also found in the insect mitogenome [1-4]. Mitogenomes have been widely used in studies regarding insect molecular systematics, phylogeography and population genetics [5-7].

Aphidinea belong to the order Hemiptera, which contains three families: Aphididae, Adelgidae and Phylloxeridae [8]. This insect group includes more than 5000 species worldwide, and the family Aphididae contains most of the species in 25 subfamilies [9]. Aphids have many intrinsically interesting characteristics, such as complex life cycles, pleomorphism and polymorphism, harboring different endosymbionts, inducing diverse galls on host plants and differentiation of social behavior [10].Therefore, all these characteristics make aphids anappropriate model for ecological and evolutionary studies [11]. 
Out of numerous aphids worldwide, only few have a complete or near-complete mitogenome currently available in GenBank (Table 1). Especially, except for the Aphidinae, the data for other subfamilies are quite rare. In 2014, we reported the mitogenome of Cervaphis quercus Takahashi (Aphididae: Greenideinae), which was the first and only mitogenome of aphids not belonging to the Aphidinae so far [12].

Table 1. The mitochondrial genomes of aphids.

\begin{tabular}{llllll}
\hline Category & Species & Length $\mathbf{( b p )}$ & GenBank No. & Reference \\
\hline \multirow{4}{*}{ Aphididae } & Schizaphis graminum & 15,721 & NC_006158 & Thao et al., 2004 [13] \\
& \multirow{4}{*}{ Aphidinae } & Acyrthosiphon pisum & 16,971 & NC_011594 & IAGC, 2010 [14] \\
& & Diuraphis noxia & 15,784 & NC_022727 & Zhang et al., 2014 [15] \\
& & Sitobion avenae & 15,180 & NC_024683 & Zhang et al., 2014 [16] \\
& & Cavariella salicicola & 16,317 & NC_022682 & Wang et al., 2013 [17] \\
& & Aphis gossypii & 15,869 & NC_024581 & Zhang et al., 2014 [18] \\
\cline { 2 - 6 } & Greenideinae & Cervaphis quercus & 15,272 & NC_024926 & Wang et al., 2014 [12] \\
\cline { 2 - 5 } & Mindarinae & Mindarus keteleerifoliae & 15,199 & KP722576 & This study \\
\hline Phylloxeridae & & Viteus vitifoliae* & 12,349 & DQ021446 & Direct submission \\
\hline
\end{tabular}

${ }^{*}$ Nearly complete.

The subfamily Mindarinae was considered a relatively age-old subfamily in Aphididae [19]. Species of this subfamily are conifer-feeding aphids, where the apterae with head fused with pronotum have three-facetted eyes, well-developed dorsal wax glands, and a tongue-shaped cauda [20].This subfamily only contains one genus, Mindarus Koch, which has extant species and eight fossil species worldwide [9]. Mindarus keteleerifoliae Zhang is a Chinese endemic species from the Hengduan Mountains [21]; and it mainly infests the leaves and young shoots of Keteleeria Carr. plants, an endemic plant group in East Asia.

Therefore, in the present study, we sequenced and annotated the complete mitogenome of $M$. keteleerifoliae, which represent the subfamily Mindarinae. Aphids of M. keteleerifoliae are harmful to old coniferous trees because they cause leaves to change color and die. Our results identified a tRNA isomer in the aphid mitogenome. Furthermore, the M. keteleerifoliae mitogenome was compared with mitogenomes from other aphids, thus increasing our understanding of aphid phylogeny and evolution.

\section{Results and Discussion}

\subsection{Genome Organization and Composition}

The complete mitogenome of M. keteleerifoliae is a double-stranded plasmid with 15,199 bp containing 13 PCGs, 22 tRNA genes, 2 rRNA genes, and a control region (Figure 1). Twenty-three genes were transcribed on the majority strand (J-strand), whereas the other fourteen were coded on the minority strand (N-strand). This mitogenome sequence was then submitted to GenBank (No. KP722576). These genes were arranged in the same order as the inferred insect ancestral mitogenome [3], Drosophila yakuba [22]. Currently, M. keteleerifoliae has the shortest mitogenome length in aphids (Table 1). The length variation was conserved in PCGs, tRNAs, $r r n S$ and $r r n L$, which was due to variation in the mainly intergenic spacers, such as the control region and repeat region $[12,15]$. In M. keteleerifoliae, 10 overlaps (a total of $33 \mathrm{bp}$ ) between adjacent genes were detected (Figure 1, Table 2). The atp8-atp6 overlap often exists in insect mitogenomes and is $7 \mathrm{bp}$ [23]. However, exceptions to this overlap were found in aphids (a 20-bp overlap in most aphids, and a 14-bp overlap between atp6 and atp8in Diuraphis noxia) [12,15]. Actually, there are many exceptions in other reported insects, such as a 19-bp overlap in the honeybee (Apis mellifera) [24] and a 244-bp long spacer in a hymenopteran (Evania appendigaster) [25]. Therefore, Lavrov's 7 bp hypothesis will be challenged with the increasing data offered by this mitogenome analysis. 


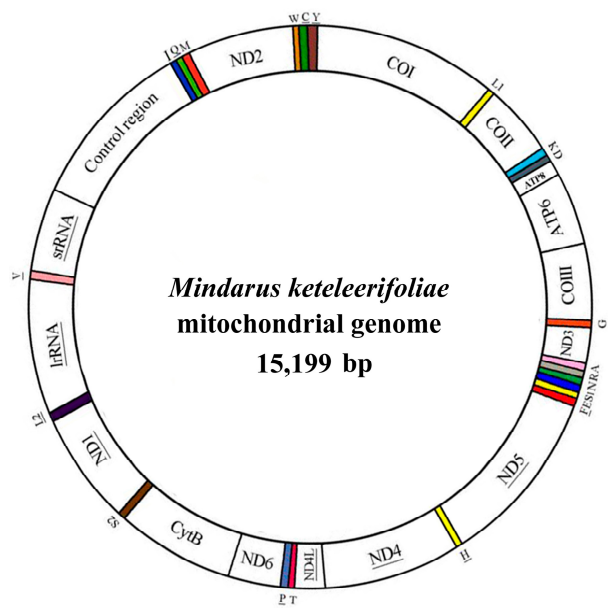

Figure 1. Circular map of the Mindarus keteleerifoliae mitogenome. Gene names not underlined indicate the direction of transcription in the major strand, and underlined names indicate the direction of transcription in the minor strand. The transfer RNAs (tRNAs) are denoted by the colored blocks and are labeled according to the single-letter amino acid codes.

Table 2. Organization of the Mindarus keteleerifoliae mitochondrial genome.

\begin{tabular}{|c|c|c|c|c|c|c|c|}
\hline Gene & Strand & Position & Anticodon & Size (bp) & Start Codon & Stop Codon & Intergenic Nucleotides * \\
\hline $\operatorname{cox} 1$ & $\mathrm{~J}$ & $1-1531$ & - & 1531 & ATA & $\mathrm{T}$ & -1 \\
\hline tRNA-Leu & $\mathrm{J}$ & $1531-1596$ & TAA & 66 & - & - & 3 \\
\hline $\operatorname{cox} 2$ & $\mathrm{~J}$ & $1600-2271$ & - & 672 & ATA & TAA & - \\
\hline$t R N A-L y s$ & $\mathrm{~J}$ & $2272-2344$ & CTT & 73 & - & - & 7 \\
\hline$t R N A-A s p$ & $\mathrm{~J}$ & $2352-2419$ & GTC & 68 & - & - & 17 \\
\hline atp8 & $\mathrm{J}$ & $2437-2586$ & - & 150 & ATA & TAA & -20 \\
\hline atp6 & $\mathrm{J}$ & $2567-3220$ & - & 654 & ATT & TAA & \\
\hline $\cos 3$ & $\mathrm{~J}$ & $3221-4006$ & - & 786 & ATG & TAA & -1 \\
\hline tRNA-Gly & $\mathrm{J}$ & $4007-4068$ & TCC & 62 & - & - & -3 \\
\hline nad3 & $\mathrm{J}$ & $4066-4422$ & - & 357 & ATA & TAA & - \\
\hline tRNA-Ala & $\mathrm{J}$ & $4423-4487$ & TGC & 65 & - & - & -1 \\
\hline$t R N A-A r g$ & $\mathrm{~J}$ & $4487-4555$ & TCG & 69 & - & - & -1 \\
\hline$t R N A-A s n$ & $\mathrm{~J}$ & $4555-4623$ & GTT & 69 & - & - & -1 \\
\hline tRNA-Ser & $\mathrm{J}$ & $4623-4684$ & TCT & 62 & - & - & 6 \\
\hline tRNA-Glu & $\mathrm{J}$ & $4691-4764$ & TTC & 74 & - & - & 3 \\
\hline tRNA-Phe & $\mathrm{N}$ & $4768-4837$ & GAA & 70 & - & - & - \\
\hline nad5 & $\mathrm{N}$ & $4838-6508$ & - & 1671 & ATT & TAA & - \\
\hline tRNA-His & $\mathrm{N}$ & $6509-6574$ & GTG & 66 & - & - & - \\
\hline nad4 & $\mathrm{N}$ & $6575-7883$ & - & 1309 & ATA & $\mathrm{T}$ & 5 \\
\hline nad4L & $\mathrm{N}$ & 7889-8179 & - & 291 & ATA & TAA & - \\
\hline$t R N A-T h r$ & $\mathrm{~J}$ & $8180-8245$ & TGT & 66 & - & - & - \\
\hline tRNA-Pro & $\mathrm{N}$ & $8246-8316$ & TGG & 71 & - & - & 1 \\
\hline nad6 & $\mathrm{J}$ & 8318-8812 & - & 495 & ATT & TAA & -1 \\
\hline$c o b$ & $\mathrm{~J}$ & $8812-9927$ & - & 1116 & ATG & TAA & -2 \\
\hline tRNA-Ser & $\mathrm{J}$ & 9926-9990 & TGA & 65 & & & 10 \\
\hline nad1 & $\mathrm{N}$ & 10001-10936 & $6-$ & 936 & ATT & TAA & - \\
\hline tRNA-Leu & $\mathrm{N}$ & $10937-11001$ & $1 \mathrm{TAG}$ & 65 & - & - & - \\
\hline$r r n L$ & $\mathrm{~N}$ & $11002-12267$ & $7-$ & 1266 & - & - & 1 \\
\hline$t R N A-\mathrm{Val}$ & $\mathrm{N}$ & $12269-12330$ & 0 TAC & 62 & - & - & 3 \\
\hline$r r n S$ & $\mathrm{~N}$ & $12334-13098$ & $8-$ & 765 & - & - & - \\
\hline $\begin{array}{l}\text { control } \\
\text { region }\end{array}$ & - & 13099-13785 & $5-$ & 687 & - & - & - \\
\hline tRNA-Ile & $\mathrm{J}$ & $13786-13851$ & 1 GAT & 66 & - & - & 3 \\
\hline$t R N A-G l n$ & $\mathrm{~N}$ & $13855-13920$ & 0 TTG & 66 & - & - & 29 \\
\hline tRNA-Met & $\mathrm{J}$ & $13950-14017$ & 7 CAT & 68 & - & - & - \\
\hline nad2 & $\mathrm{J}$ & 14018-14995 & $5-$ & 978 & ATA & TAA & - \\
\hline$t R N A-\operatorname{Tr} p$ & $\mathrm{~J}$ & $14996-15063$ & 3 TCA & 68 & - & - & -2 \\
\hline tRNA-Cys & $\mathrm{N}$ & $15062-15127$ & 7 GCA & 66 & - & - & 4 \\
\hline$t R N A-T y r$ & $\mathrm{~N}$ & 15132-15198 & 8 GTA & 67 & - & - & 1 \\
\hline
\end{tabular}


The nucleotide composition of $M$. keteleerifoliae is typically $\mathrm{A}+\mathrm{T}$ biased with $84.52 \%$ and is slightly A skewed (0.06) and strongly C skewed (-0.23) (Supplementary Material, Table S1). Similar patterns of nucleotide composition were also found in other aphid species and gathered into a cluster [26], representing high $\mathrm{A}+\mathrm{T}$ content in Hemipteran species. Analysis of the base composition at each codon position of the concatenated 13 PCGs suggested that the A+T content of all codon positions is above $80 \%$ (Supplementary Material, Table S1). In the first codon position, the strongest bias toward T was $(48.0 \%)$, while the third codon position had the highest $\mathrm{G}+\mathrm{C}$ content (19.0\%). Actually, aphids with $\mathrm{A}+\mathrm{T}$ rich and G-deficit mitogenomes were similar to other insects [3]. GC content strongly determines codon bias as well as mutational selection and pressure at the genome level in the prevailing theories of neutral mutations[27].The low GC content found in aphid population genetic studies may indicate a potential explanation for the slow mutation rates observed commonly in aphid mtDNA sequences [28,29].

\subsection{Protein-Coding Genes}

The mitogenome of $M$. keteleerifoliae includes the 13 PCGs that are present in animal mitogenomes and their arrangement orders are similar to the standard order of insect [3]. In the M. keteleerifoliae mitogenome, the start codon is one of the typical ATN codons, four (atp6, nad1, nad5 and nad6) with ATT, two (coband cox3) with ATG, and the remainder with ATA (Table 2). The most common TAA termination codons were detected in 11 PCGs (cox2, cox3, cob, atp6, atp8, nad1, nad2, nad3, nad4L, nad5 and nad6). Cox1 and nad4, the remaining two, had incomplete termination codons with T (Table 2). In the mitogenomes of most insects, partial stop codons are common [1], including the currently sequenced aphid species $[12,15,17]$.

There are 3637 amino-acid-coding codons that were calculated for codon usage of the $M$. keteleerifoliae mitogenome. Approximately the same codon numbers were found in Acyrthosiphon pisum (Harris) (3637) and Schizaphis graminum (Rondani) (3638). The five most abundant codons are UUU (Phe), UUA (Leu), AUU (Ile), AUA (Met) and AAU (Asn) (Supplementary Material, Table S2), and reflect the $A+T$ bias. Cystine is the least frequent as in other aphids [12,15]. The third codon positions have the strongest $\mathrm{A}+\mathrm{T}$ bias (Supplementary Material, Table S2).

\section{3. $t R N A$ and $r R N A$}

In the M. keteleerifoliae mitogenome (Table 2), all 22 typical animal tRNA genes with length from 62 to $74 \mathrm{bp}$ were found, and 20 were determined using the tRNAscan-SE [16]. By comparison with currently published aphid mitogenomes, the tRNA-Asn and tRNA-Ser(AGN) genes were determined $[12,13,15,17]$. The typical clover-leaf structure was predicted in only 21 of the 22 mitochondrial tRNAs since the $t R N A$-Ser(AGN) gene included a DHU replacement loop instead of the typical DHU arm, and could not form a stem-loop structure in the TYC arm (Figure 2). In many arthropod mitogenomes, this is a common feature [1].

Based on the secondary structure, in the M. keteleerifoliae tRNAs, a total of $13 \mathrm{G}-\mathrm{U}$ weak base pairs were found, which form weak bonds, located in the AA stem (2 bp), the T stem (1 bp) and the DHU stem (10 bp) (Figure 2). Most of the mismatched nucleotides were G-U pairs, which form weak bonds in tRNAs and non-canonical pairs in tRNA secondary structures similarly to other aphids $[12,17]$.

The boundaries of rRNA genes were implemented from the alignment with other aphid species $[12,13,17]$. The $r r n L$ of M. keteleerifoliae was located between $t R N A-L e u(C \mathrm{UN})$ and $t R N A-V a l$, and $r r n S$ resided between $t R N A$-Val and the control region similarly to other insects (Figure 1). The large ribosomal gene $(r r n L)$ of M. keteleerifoliaewas1266 bp, and has an AT content of $85.6 \%$. The small ribosomal gene ( $\mathrm{rrnS}$ ) was $765 \mathrm{bp}$, and has an AT content of $84.4 \%$ (Supplementary Material, Table S1). The identified AT contents were same to those reported species in other hemipterans [30,31]. 

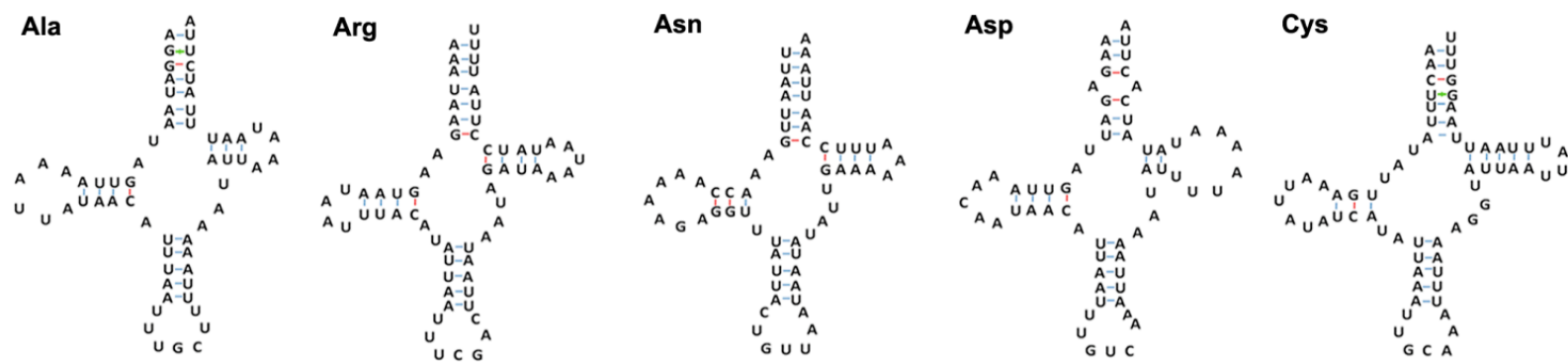

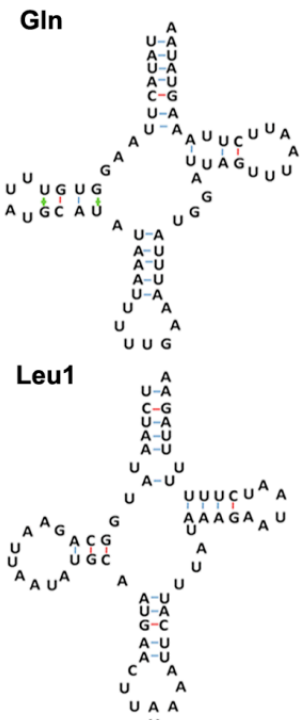

Glu

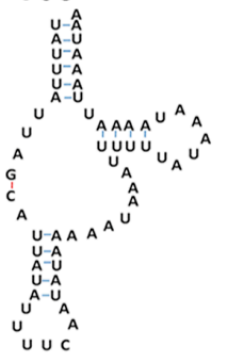

Leu2

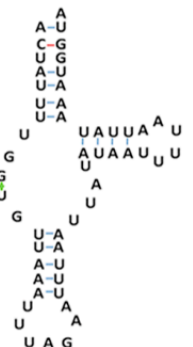

Phe

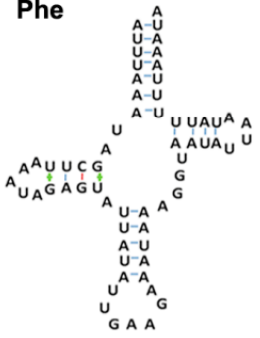

Ser1
(AGN)

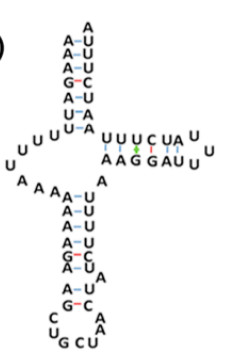

Gly

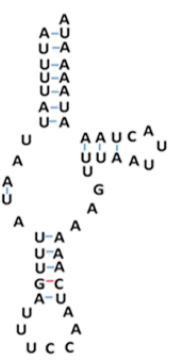

Lys

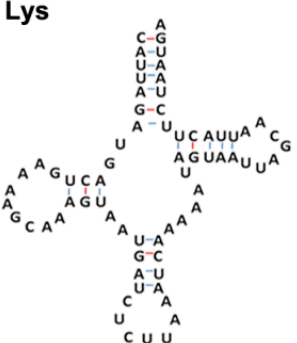

Ser2

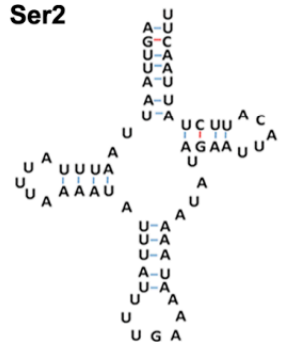

His

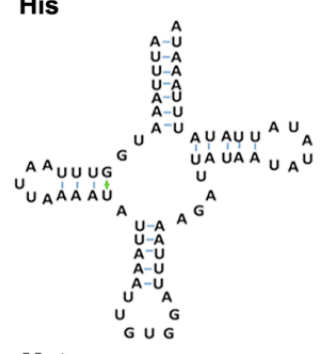

Met

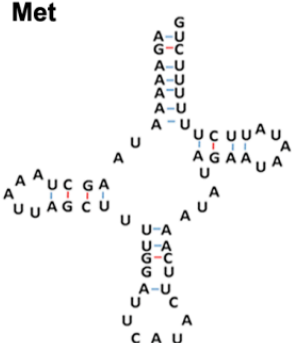

Thr

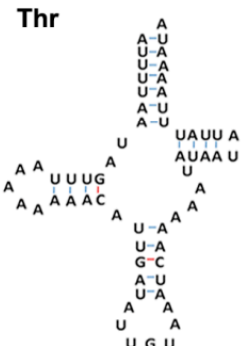

Ile
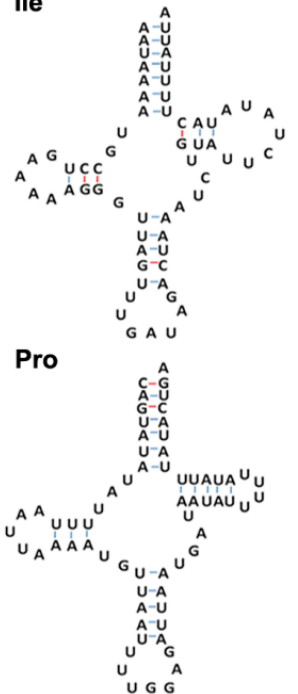

Trp

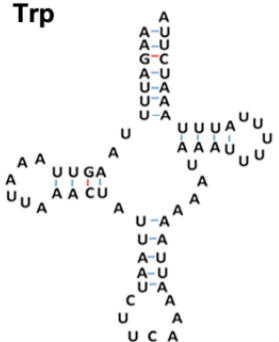

Tyr

Val

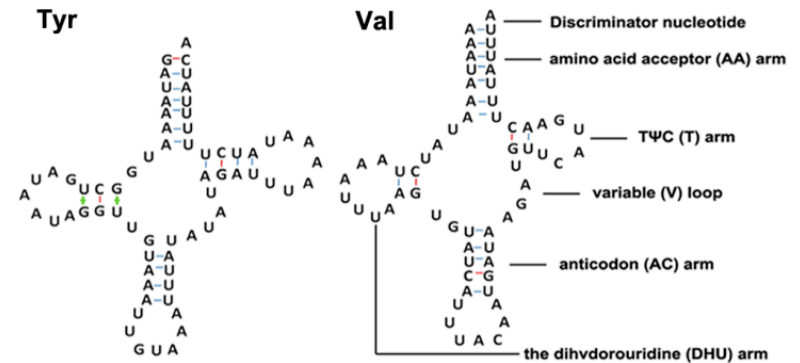

Figure 2. The inferred secondary structure of the 22 transfer RNAs (tRNAs) in the Mindarus keteleerifoliae mitogenome. The tRNAs are labeled with the abbreviations of their corresponding amino acids. Dashed line (-) indicates Watson-Crick base pairing and (+) indicates $\mathrm{G}-\mathrm{U}$ base pairing.

\section{4. tRNA Isomerism}

A tRNA isomer may be discovered in the mitogenome of $M$. keteleerifoliae. The $t R N A-T r p$ gene is coded in the J-strand; however, the same sequence in the N-strand codes for the tRNA-Ser gene (Figure 3). This phenomenon was also found in the mitogenome of Eriosoma lanigerum (our unpublished data): the $t R N A$-Gly gene and $t R N A$-Ser occurred as atRNA isomer (Figure 4). 


\section{Mindarus keteleerifoliae}

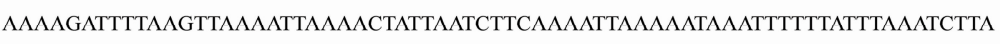

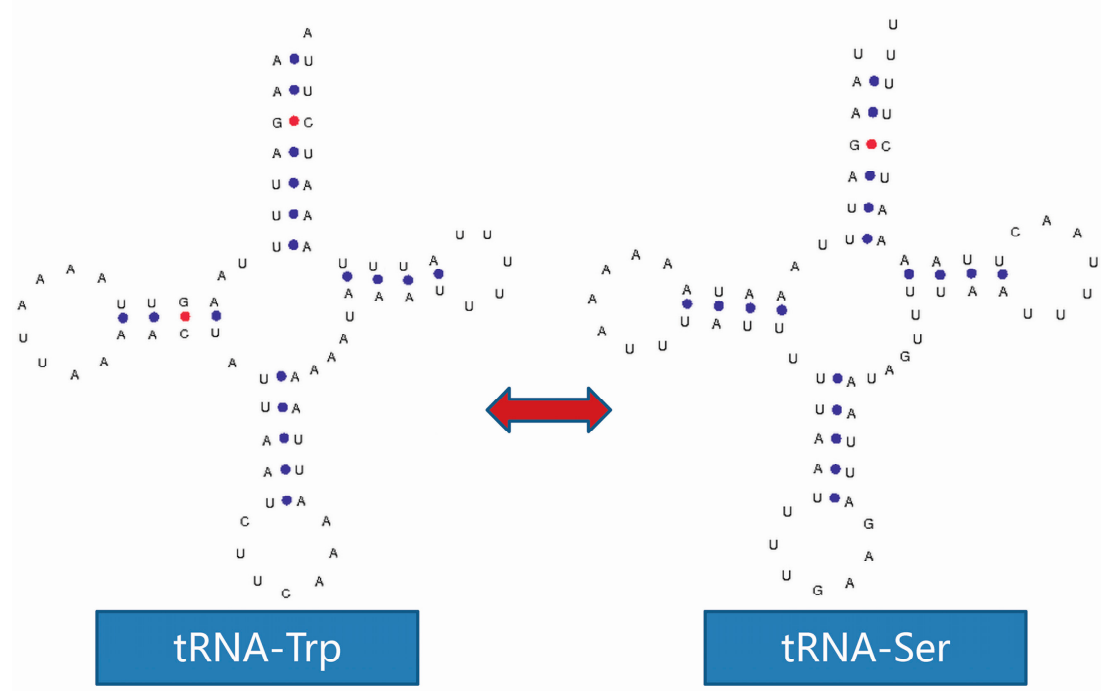

Figure 3. The tRNA isomer of Mindarus keteleerifoliae. The blue and red dots indicate Watson-Crick base pairing.

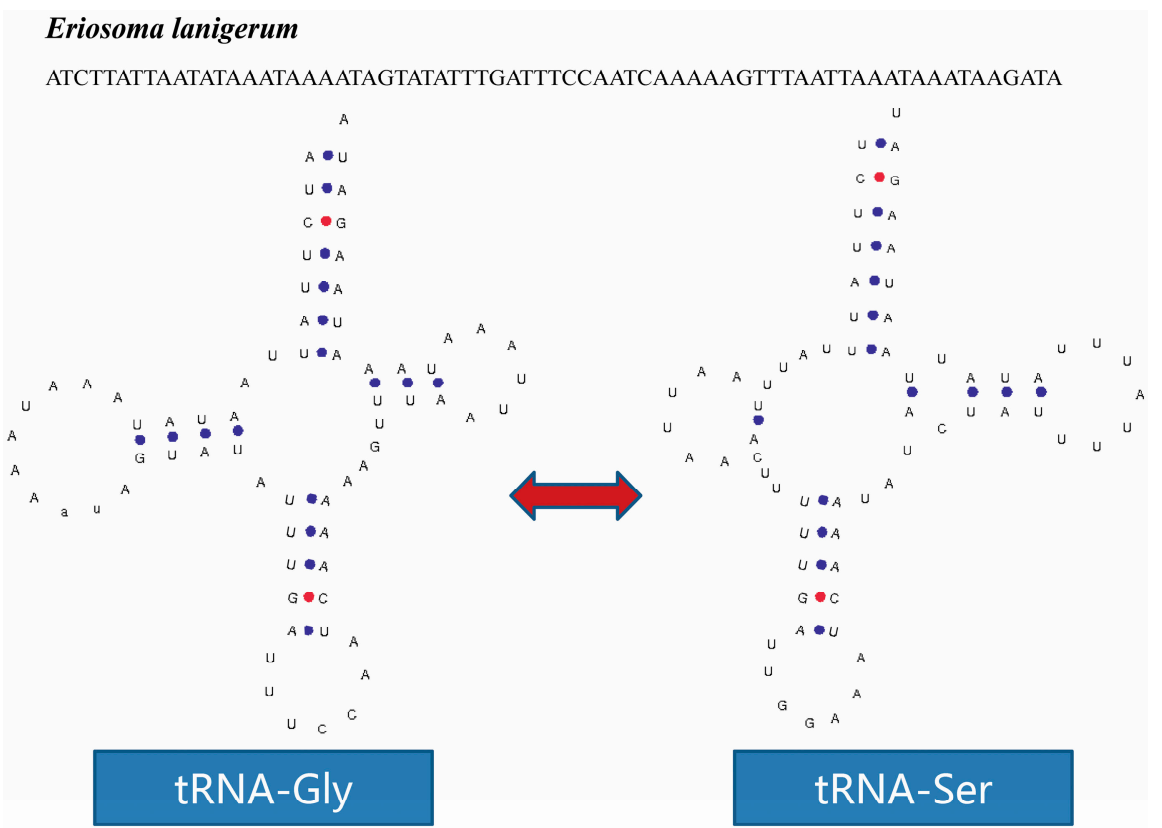

Figure 4. The tRNA isomer of Eriosoma lanigerum. The blue and red dots indicate Watson-Crick base pairing.

Therefore, both tRNAs were $t R N A-X$ and $t R N A$-Ser isomers. First, the spatial structure of the two tRNA isoforms was stable in terms of chemical free energy. However, in the tRNA-Ser gene of $M$. keteleerifoliae, the acceptor arm lost a pair of hydrogen bonds; and in the tRNA-Ser gene of Eriosoma lanigerum (Hausmann), the D loop and TYC loop arms lost two pairs of hydrogen bonds. The number of hydrogen bonds retained a low free energy and maintained stable spatial structure, leading to the formation of the typical cloverleaf secondary structure. Second, from the four types of amino acid codons involved in the two isomer phenomenon, the codons UCA and UGA had relatively higher usage frequencies in M. keteleerifoliae (Supplementary Material, Table S2); the usage frequency the codon UCC in Eriosoma lanigerum was relatively weak but not zero, and the frequency for GGA was 
relatively higher. After analyzing these two points, the tRNA isomers in aphids may exist (rather than resulting from the coincidence of sequencing results). These predicted tRNA structures cannot discern their function, but several studies have confirmed that tRNA isomers exist and are functional [32]. In aphids, determining whether the tRNA isomers exist also need further study.

\subsection{Non-Coding Regions}

Sixteen non-coding regions, with a total of $770 \mathrm{bp}$, were interspersed throughout the M. keteleerifoliae mitogenome (Table 2). As in typical insect mitogenomes, the M. keteleerifoliae mitogenome includes one large non-coding region, identified as the control region.

In M. keteleerifoliae, the control region was rich in $\mathrm{A}+\mathrm{T}(88.9 \%)$ and located downstream of $r r n S$. This region commonly contains replication origins in both invertebrates and vertebrates $[2,3]$. Meanwhile, the control region had a higher $\mathrm{A}+\mathrm{T}$ content than the whole majority strand of all reported aphid mitogenomes $[12,15,17]$. The lengths of the control regions in aphid mitogenomes are variable, as shown by the control region of $M$. keteleerifoliae with a length of $687 \mathrm{bp}$, which is approximately half the length of A. pisum (1336 bp)and Cavariella salicicola (Matsumura) (1137 bp), although similar to C. quercus (657 bp), S. graminum (682 bp) and Diuraphis noxia (Kurdjumov) (664 bp). These differences may cause their various structural patterns. Only two species of Aphidinae contained tandem repeat sequences: A. pisum and C. salicicola[17]. The control regions of A. pisum and C. salicicola can be divided into four parts (Figure 5): a region composed of complete tandem repeats and a partial copy of the anterior repeat unit; an $\mathrm{A}+\mathrm{T}$ rich zone; a conserved PolyT stretch; and a stem-loop region at the end of the control region $[12,17]$. However, the control regions of other aphid species also have the conserved structural pattern found in M. keteleerifoliae with three parts (Figure 5): the AT-rich region, the stem-loop structure, and the PolyT stretch regions, which were proposed as a widespread feature in Aphididae [12].

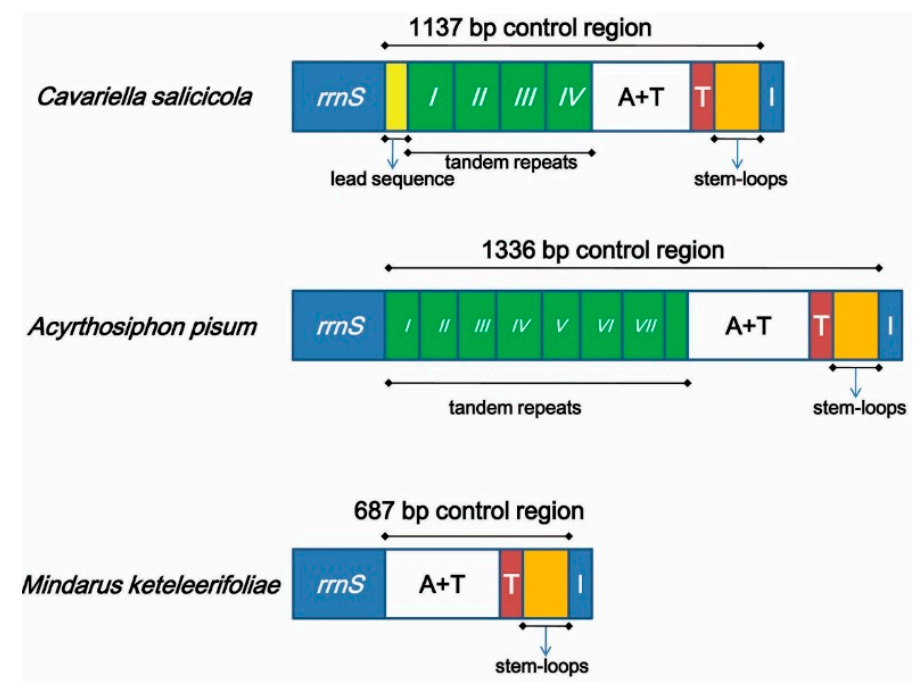

Figure 5. Control region organization in aphid mitogenomes. The lead region is in a yellow box; the green boxes with Roman numerals indicate the tandem repeat units; $\mathrm{A}+\mathrm{T}$ represents a high $\mathrm{A}+\mathrm{T}$ content region; red boxes refer to the poly-thymidine stretch; orange boxes indicate the stem-loop region.

The repeat region between $\operatorname{trnE}$ and $t r n F$ is an interesting feature of the aphid mitogenomes [17]. This unique region contains serially variable numbers of tandem repeats, but this repeat region was not found in the mitogenome of $M$. keteleerifoliae. This new evidence implies that the repeat region may be typical for Aphidinae but not of other subfamilies in Aphididae [12]. So, we thought that the repeat region within Aphidinae is lineage specific and occurred from independent evolutionary events. 


\subsection{Phylogenetic Analyses}

For the phylogenetic analysis, the newly sequenced M. keteleerifoliae was combined with the mitogenome sequences of seven aphid species. The phylogenetic trees generated from ML analyses and Bayesian inferences showed similar topologies (Figure 6). The monophyly of Aphidinae, Greenideinae and Mindarinae was recovered in different analyses and well supported. M. keteleerifoliae was used as the representative of Mindarinae and located in the basal position of the Aphididae. This result suggests that the Mindarinae aphids are the ancient species of Aphididae and were conifer feeders able to retain an ancestral host relationship with gymnosperms [33]. Meanwhile, some evidence of fossil characteristics also supported the position of Mindarinae [19,34]. Within the Aphidinae subfamily, the monophyly of Aphidini and Macrosiphini had statistically high values, and is similar to the traditional taxonomic views based on morphology [35], and the results based on previous molecular phylogenetic studies [36,37].

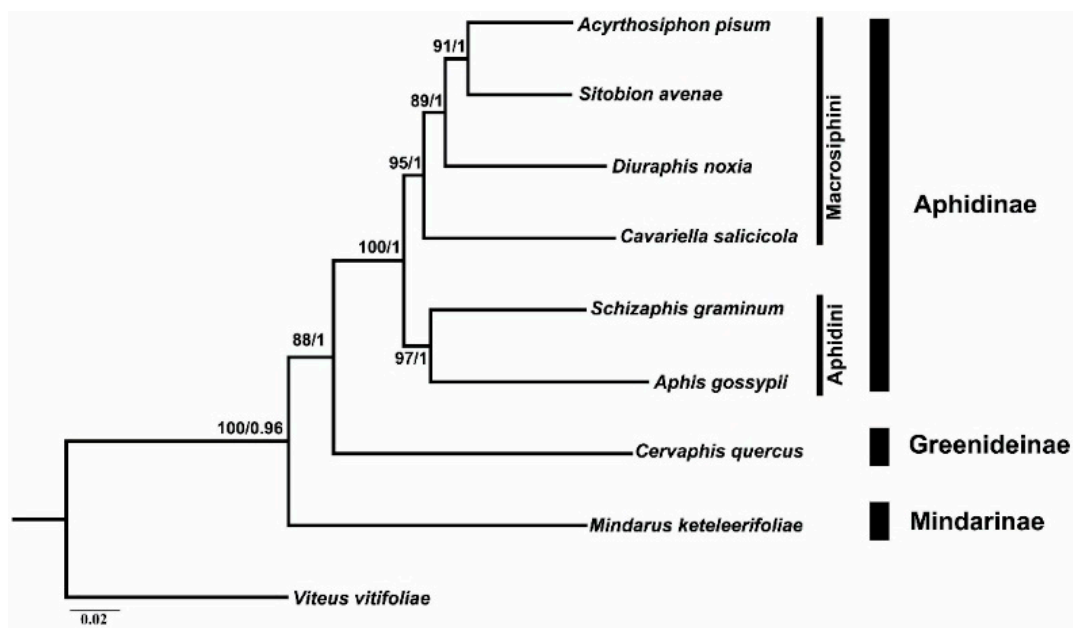

Figure 6. ML and BI Phylogenetic tree inferred from aphid mitogenome sequences. The node support values are the bootstrap (BS) values and the Bayesian posterior probabilities (BPP).

\section{Materials and Methods}

\subsection{Experimental Sample}

About 50 individuals of M. keteleerifoliae were collected from Kunming, Yunnan Province in 2006. The specimens for slide mounting were preserved in 75\% ethanol, and samples for molecular research were stored in $95 \%$ ethanol at $-20{ }^{\circ} \mathrm{C}$ before the DNA extraction. Specimen examination was conducted by Gexia Qiao with a Leica microscope (Leica DM2500, Wetzlar, Germany) based on identified specimens, monographs [21], and the original morphological descriptions. All voucher specimens and samples were deposited in the National Zoological Museum of China at the Institute of Zoology, Chinese Academy of Sciences, Beijing, China (NZMCAS).

\subsection{DNA Extraction, Amplification and Sequencing}

Using the DNeasy Tissue Kit (QIAGEN, Hilden, Germany), total DNA was extracted from single aphids preserved in $95 \%$ ethanol following the protocols. Short and long PCR cycles were used to amplify the whole mitogenome of $M$. keteleerifoliae. Short amplicons reactions were amplified using universal primers (some modified), and long amplicons were amplified using specific primers according to the fragments of the short amplicons similar to our previous study [12]. The primers were designed according to the conserved regions by the program Primer premier 5.0. In this study, all the primers used were synthesized by Invitrogen Biotech (Beijing, China) and are listed in Table S3 [38-41]. Short amplicons (sequence length $<1.5 \mathrm{k}$ ) were amplified at the following settings with Taq DNA 
polymerase (TransGen Biotech, Beijing, China): $95^{\circ} \mathrm{C}$ for $3 \mathrm{~min}$; 35 cycles of $92^{\circ} \mathrm{C}$ for $1 \mathrm{~min}, 48-55^{\circ} \mathrm{C}$ (depending on primer pairs) for $1 \mathrm{~min}$ and $72^{\circ} \mathrm{C}$ for $2 \mathrm{~min}$. A final extension step of $10 \mathrm{~min}$ was at $72^{\circ} \mathrm{C}$ after cycling. Long amplicons (sequence length $>1.5 \mathrm{k}$ ) were amplified under the following cycling conditions with the High Fidelity (HiFi) Taq DNA polymerase (TransGen Biotech, Beijing, China): $2 \mathrm{~min}$ at $92^{\circ} \mathrm{C}, 10$ cycles $\left(10 \mathrm{~s}\right.$ at $92^{\circ} \mathrm{C}, 30 \mathrm{~s}$ at $50-55^{\circ} \mathrm{C}$ (depending on primer pairs), and $4-8 \mathrm{~min}$ at $\left.68^{\circ} \mathrm{C}\right), 20$ cycles $\left(10 \mathrm{~s}\right.$ at $92^{\circ} \mathrm{C}, 30 \mathrm{~s}$ at $50-55^{\circ} \mathrm{C}$, and $4-8$ min at $68^{\circ} \mathrm{C}$ with an additional $30 \mathrm{~s}$ per cycle), and a final prolonged elongation of $10 \mathrm{~min}$ at $72{ }^{\circ} \mathrm{C}$. PCR products were detected by electrophoresis on a $1 \%$ agarose gel and purified using the EasyPure PCR purification Kit (TransGen Biotech, Beijing, China). All short PCR fragments were directly sequenced for both strands. Long PCR fragments were cloned into the pMD19-T sequencing vector (TaKaRa, Dalian, China) and sequenced using the primer walking strategy. Sequencing reactions were implemented by the BigDye Terminator v3.1 Cycle Sequencing Kit (Applied Biosystems, Foster City, CA, USA) and run on an ABI 3730 automated sequencer (Applied Biosystems).

\subsection{Mitogenome Annotation and Analysis}

All individuals are from the same clone, and the genetic variation was not found between isolates. So, one mitogenome was assembled. Sequences were assembled by the software SeqMan (DNAStar Inc., Madison, WI, USA). Sequence annotation was implemented using the blast tools on the NCBI web site [42]. The 13 PCGs and two ribosomal RNA genes were identified by sequence similarity with some published aphid mitogenomes (A. pisum NC_011594.1, C. salicicola NC_022682, D. noxia NC_022727 and S. graminum NC_006158.1). The nucleotide sequences of the PCGs were translated with the invertebrate mitogenome genetic code. The tRNAs were predicted by the tRNAscan-SE Search Server v.1.21 [43] using the default settings. Two tRNA genes ( $t R N A-A s n$ and $t R N A-\operatorname{Ser}(\mathrm{AGN}))$ were not found by the tRNAscan-SE, but were identified by comparison with other aphids and edited by eyes. The $\mathrm{A}+\mathrm{T}$ content and codon usage were calculated by the MEGA version 6.05 [44]. Strand asymmetry was calculated using the formulas GC skew $=(\mathrm{G}-\mathrm{C}) /(\mathrm{G}+\mathrm{C})$ and AT skew $=(\mathrm{A}-\mathrm{T}) /(\mathrm{A}+\mathrm{T})[45]$ for the strand encoding the majority of the protein-coding genes. The putative control region was detected for regions of potentially palindromes or inverted repeats with the Mfold web server [46].

\subsection{Phylogenetic Analysis}

For M. keteleerifoliae, one individual was used for molecular analysis. The multiple alignments of the concatenated 13 PCG nucleotide sequences for the 9 aphid mitogenomes, including 8 Aphididae species and one Phylloxeridae species as the outgroup (Table 1), were conducted with the MEGA version 6.05 [44] and then manually proofread. Alignments of individual genes were then concatenated after excluding the stop codons.

Maximum likelihood (ML) and Bayesian inference (BI) analyses were calculated using PHYML 3.0 [47] and MrBayes version 3.1.2 [48], respectively. The JModelTest 3.7 was implemented to select an appropriate nucleotide substitution model [49]. The GTR+I+G was the optimal model by the Bayesian information criterion (BIC) [50]. ML analyses were under the optimal substitution model from the JModelTest, and model parameter values were valued during the analyses. Nodal support of branches was evaluated by bootstrap analysis with 100 replicates. The Bayesian inference used two independent runs with 10,000,000 generations in each and four chains. Each chain was sampled every 1000 generations with a burn-in of $25 \%$. Trees inferred prior to stationary were discarded as burn-in, and the remaining trees were constructed using a 50\% majority-rule consensus tree with posterior probabilities.

\section{Conclusion}

This paper reports the complete mitogenome of the aphidM. keteleerifoliae, and compares the analysis to published aphid mitochondrial genomes. The results suggest that gene size, content, and base compositions are similar among Aphididae mitogenomes. Most tRNAs are folded into the classic 
clover-leaf structure, with the exception of $t R N A$-Ser (AGN). A tRNA isomer maybe identified in the mitogenomes of $M$. keteleerifoliae and E. lanigerum; whetherthe tRNA isomers in aphids exist needs further study. Phylogenetic reconstructions based on protein-coding genes showed Mindarinae at the basal position of Aphididae. Two tribes of Aphidinae were recovered with high statistical significance. Therefore, because only eight complete aphid mitogenomes were published, including M. keteleerifoliae, future studies with larger taxon sampling sizes are necessary.

Supplementary Materials: Supplementary materials can be found at http://www.mdpi.com/1422-0067/16/12/ $26219 / \mathrm{s} 1$.

Acknowledgments: We thank Yaqiong Li and Fangfang Niu for some previous data analyses, Xiaolei Huang and Ruiling Zhang for collecting the specimens of M. keteleerifoliae, and Fendi Yang for preparing aphid slides. The work was supported by National Natural Sciences Foundation of China (Nos. 31430078), the Ministry of Science and Technology of the People's Republic of China (MOST Grant Nos. 2011FY120200, 2014FY210200, 2013FY111200), and National Science Fund for Fostering Talents in Basic Research (No. J1210002).

Author Contributions: All authors contributed to writing this article.

Conflicts of Interest: The authors declare no conflict of interest.

\section{References}

1. Wolstenholme, D.R. Animal mitochondrial DNA: Structure and evolution. Int. Rev. Cytol. 1992, 141, $173-216$. [PubMed]

2. Zhang, D.X.; Hewitt, G.M. Insect mitochondrial control region: A review of its structure, evolution and usefulness in evolutionary studies. Biochem. Syst. Ecol. 1997, 25, 99. [CrossRef]

3. Boore, J.L. Animal mitochondrial genomes. Nucleic Acids Res. 1999, 27, 1767-1780. [CrossRef] [PubMed]

4. Cameron, S.L. Insect mitochondrial genomics: Implications for evolution and phylogeny. Annu. Rev. Entomol. 2014, 59, 95-117. [CrossRef] [PubMed]

5. Hua, J.M.; Li, M.; Dong, P.Z.; Cui, Y.; Xie, Q.; Bu, W.J. Comparative and phylogenomic studies on the mitochondrial genomes of Pentatomomorpha (Insecta: Hemiptera: Heteroptera). BMC Genom. 2008, 9, 610. [CrossRef] [PubMed]

6. Li, H.; Liu, H.Y.; Song, F.; Shi, A.M.; Zhou, X.G.; Cai, W.Z. Comparative mitogenomic analysis of damsel bugs representing three tribes in the family Nabidae (Insecta: Hemiptera). PLoS ONE 2012, 7. [CrossRef] [PubMed]

7. Ma, C.; Yang, P.C.; Jiang, F.; Shali, Y.; Chapuis, M.P.; Sword, G.; Kang, L. Mitochondrial genomes reveal the global phylogeography and dispersal routes of the migratory locust. Mol. Ecol. 2012, 21, 4344-4358. [CrossRef] [PubMed]

8. Remaudière, G.; Remaudière, M. Catalogue des Aphididae du Monde; Institut National de la Recherche Agronomique: Paris, France, 1997.

9. Version 5.0/5.0. Available online: http://Aphid.SpeciesFile.org (accessed on 8 October 2015).

10. Blackman, R.L.; Eastop, V.F. Aphids on the World's Crops: An Identification and Information Guide, 2nd ed.; John Wiley \& Sons: Chichester, UK, 2000.

11. Huang, X.L.; Qiao, G.X. Aphids as models for ecological and evolutionary studies. Insect. Sci. 2014, 21, 247-250. [CrossRef] [PubMed]

12. Wang, Y.; Huang, X.L.; Qiao, G.X. The complete mitochondrial genome of Cervaphis quercus (Insecta: Hemiptera: Aphididae: Greenideinae). Insect. Sci. 2014, 21, 278-290. [CrossRef] [PubMed]

13. Thao, M.L.; Baumann, L.; Baumann, P. Organization of the mitochondrial genomes of whiteflies, aphids, and psyllids (Hemiptera, Sternorrhyncha). BMC Evol. Biol. 2004, 4, 25. [CrossRef] [PubMed]

14. The International Aphid Genomics Consortium. Genome sequence of the pea aphid Acyrthosiphon pisum. PLoS Biol. 2010, 8, e1000313.

15. Zhang, B.; Ma, C.; Edwards, O.; Fuller, S.; Kang, L. The mitochondrial genome of the Russian wheat aphid Diuraphis noxia: Large repetitive sequences between $\operatorname{trn} E$ and $\operatorname{trnF}$ in aphids. Gene 2014, 533, 253-260. [CrossRef] [PubMed]

16. Zhang, B.; Zheng, J.; Liang, L.; Fuller, S.; Ma, C.S. The complete mitochondrial genome of Sitobion avenae (Hemiptera: Aphididae). Mitochondrial DNA 2014. [CrossRef] 
17. Wang, Y.; Huang, X.L.; Qiao, G.X. Comparative analysis of mitochondrial genomes of five aphid species (Hemiptera: Aphididae) and phylogenetic implications. PLoS ONE 2013, 8. [CrossRef] [PubMed]

18. Zhang, S.; Luo, J.; Wang, C.; Lv, L.; Li, C.; Jiang, W.; Cui, J.; Rajput, L.B. Complete mitochondrial genome of Aphis gossypii Glover (Hemiptera: Aphididae). Mitochondrial DNA 2014. [CrossRef]

19. Heie, O.E. Paleontology and phylogeny. In Aphids: Their Biology, Natural Enemies and Control; Elsevier: Amsterdam, The Netherlands, 1987.

20. Quednau, F.W. Atlas of the Drepanosiphine Aphids of the World Part III; The American Entomological Institute: Gainesville, FL, USA, 2010.

21. Zhang, G.X.; Qiao, G.X.; Zhong, T.S.; Zhang, W.Y. Homoptera: Mindaridae and Pemphigidae; Science Press: Beijing, China, 1999.

22. Clary, D.O.; Wolstenholme, D.R. The mitochondrial DNA molecular of Drosophila yakuba: Nucleotide sequence, gene organization and genetic code. J. Mol. Evol. 1985, 22, 252-271. [CrossRef] [PubMed]

23. Lavrov, D.V.; Boore, J.L.; Brown, W.M. The complete mitochondrial DNA sequence of the horseshoe crab Limulus polyphemus. Mol. Biol. Evol. 2000, 17, 813-824. [CrossRef] [PubMed]

24. Gillespie, J.J.; Johnston, J.S.; Cannone, J.J.; Gutell, R.R. Characteristics of the nuclear (18S, 5.8S, 28S and 5S) and mitochondrial (12S and 16S) rRNA genes of Apis mellifera (Insecta: Hymenoptera): Structure, organization and retrotransposable elements. Insect Mol. Biol. 2006, 15, 657-686. [CrossRef] [PubMed]

25. Wei, S.J.; Shi, M.; Sharkey, M.J.; van Achterberg, C.; Chen, X.X. Comparative mitogenomics of Braconidae (Insecta: Hymenoptera) and the phylogenetic utility of mitochondrial genomes with special reference to holometabolous insects. BMC Genom. 2010, 11, 371. [CrossRef] [PubMed]

26. Wang, Y.; Chen, J.; Jiang, L.Y.; Qiao, G.X. Hemipteran mitochondrial genomes: Features, structures and implications for phylogeny. Int. J. Mol. Sci. 2015, 16, 12382-12404. [CrossRef] [PubMed]

27. Hershberg, R.; Petrov, D.A. Selection on codon bias. Annu. Rev. Genet. 2008, 42, 287-299. [CrossRef] [PubMed]

28. Peccoud, J.; Ollivier, A.; Plantegenest, M.; Simon, J.C. A continuum of genetic divergence from sympatric host races to species in the pea aphid complex. Proc. Natl. Acad. Sci. USA 2009, 106, 7495-7500. [CrossRef] [PubMed]

29. Zhang, B.; Edwards, O.R.; Kang, L.; Fuller, S.J. Russian wheat aphids (Diuraphis noxia) in China: Native range expansion or recent introduction? Mol. Ecol. 2012, 21, 2130-2144. [CrossRef] [PubMed]

30. Lee, W.; Kang, J.; Jung, C.; Hoelmer, K.; Lee, S.; Lee, S. Complete mitochondrial genome of brown marmorated stink bug Halyomorpha halys (Hemiptera: Pentatomidae), and phylogenetic relationships of hemipteran suborders. Mol. Cell 2009, 28, 155. [CrossRef] [PubMed]

31. Li, H.; Gao, J.; Liu, H.; Liang, A.; Zhou, X.; Cai, W.Z. The architecture and complete sequence of mitochondrial genome of an assassin bug Agriosphodrus dohrni (Hemiptera: Reduviidae). Int. J. Biol. Sci. 2011, 7, 792. [CrossRef] [PubMed]

32. Sakurai, M.; Watanabe, Y.; Watanabe, K.; Ohtsuki, T. A protein extension to shorten RNA: Elongated elongation factor-Tu recognizes the D-arm of T-armless tRNAs in nematode mitochondria. Biochem. J. 2006, 399, 249-256. [CrossRef] [PubMed]

33. Von Dohlen, C.D.; Moran, N.A. Molecular data support a rapid radiation of aphids in the Cretaceous and multiple origins of host alternation. Biol. J. Linn. Soc. 2000, 71, 689-717. [CrossRef]

34. Heie, O.E.; Wegierek, P. A classification of the Aphidomorpha (Hemiptera: Sternorrhyncha) under consideration of the fossil taxa. Redia 2009, 92, 69-77.

35. Heie, O.E. The aphidoidea (hemiptera) of fennoscandia and Denmark. IV. In Family Aphididae: Part 1 of Tribe Macrosiphini of Subfamily Aphidinae; E.J. Brill/Scandinavian Science Press Ltd.: Leiden, The Netherlands, 1992.

36. Ortiz-Rivas, B.; Martínez-Torres, D. Combination of molecular data support the existence of three main lineages in the phylogeny of aphids (Hemiptera: Aphididae) and the basal position of the subfamily Lachninae. Mol. Phylogenet. Evol. 2010, 55, 305-317. [CrossRef] [PubMed]

37. Kim, H.; Lee, S.; Jang, Y. Macroevolutionary patterns in the Aphidini aphids (Hemiptera: Aphididae): Diversification, host association, and biogeographic origins. PLOS ONE 2011, 6. [CrossRef] [PubMed]

38. Foottit, R.G.; Maw, H.E.L.; Von Dohlen, C.D.; Hebert, P.D.N. Species identification of aphids (Insecta: Hemiptera: Aphididae) through DNA barcodes. Mol. Ecol. Res. 2008, 8, 1189-1201. [CrossRef] [PubMed] 
39. Normark, B.B. Phylogeny and evolution of parthenogenetic weevils of the Aramigus tessellatus species complex (Coleoptera: Curculionidae: Naupactini): Evidence from mitochondrial DNA sequences. Evolution 1996, 50, 734-745. [CrossRef]

40. Simon, C.; Buckley, T.R.; Frati, F.; Stewart, J.B.; Beckenbach, A.T. Incorporating molecular evolution into phylogenetic analysis, and a new compilation of conserved polymerase chain reaction primers for animal mitochondrial DNA. Annu. Rev. Ecol. Evol. Syst. 2006, 37, 545-579. [CrossRef]

41. Simon, C.; Frati, F.; Beckenbach, A.T.; Crespi, B.; Liu, H.; Flook, P. Evolution, weighting, and phylogenetic utility of mitochondrial gene sequences and a compilation of conserved polymerase chain reaction primers. Ann. Entomol. Soc.Am. 1994, 87, 651-701. [CrossRef]

42. NCBI Web Site. Available online: http://blast.ncbi.nlm.nih.gov/Blast (accessed on 8 October 2015).

43. Lowe, T.M.; Eddy, S.R. tRNAscan-SE: A program for improved detection of transfer RNA genes in genomic sequence. Nucleic Acids Res. 1997, 25, 955-964. [CrossRef] [PubMed]

44. Tamura, K.; Stecher, G.; Peterson, D.; Filipski, A.; Kumar, S. MEGA6: Molecular evolutionary genetics analysis version 6.0. Mol. Biol. Evol. 2013, 30, 2725-2729. [CrossRef] [PubMed]

45. Perna, N.T.; Kocher, T.D. Patterns of nucleotide composition at fourfold degenerate sites of animal mitochondrial genomes. J. Mol. Evol. 1995, 41, 353-358. [CrossRef] [PubMed]

46. Mfold Web Server. Available online: http://www.bioinfo.rpi.edu/applications/mfold/ (accessed on 8 October 2015).

47. Guindon, S.; Gascuel, O. A simple, fast, and accurate algorithm to estimate large phylogenies by maximum likelihood. Syst. Biol. 2003, 52, 696-704. [CrossRef] [PubMed]

48. Ronquist, F.; Huelsenbeck, J.P. MrBayes 3: Bayesian phylogenetic inference under mixed models. Bioinformatics 2003, 19, 1572-1574. [CrossRef] [PubMed]

49. Posada, D. jModelTest: Phylogenetic model averaging. Mol. Biol. Evol. 2008, 25, 1253-1256. [CrossRef] [PubMed]

50. Schwarz, G. Estimating the dimension of a model. Ann. Stat. 1978, 6, 461-464. [CrossRef]

(C) 2015 by the authors; licensee MDPI, Basel, Switzerland. This article is an open access article distributed under the terms and conditions of the Creative Commons by Attribution (CC-BY) license (http://creativecommons.org/licenses/by/4.0/). 